\title{
Fixed point analysis for multi-valued operators with graph approach by the generalized Hausdorff distance
}

\author{
Marwan Amin Kutbi ${ }^{1}$ and Wutiphol Sintunavarat ${ }^{2}$
}

\author{
"Correspondence: \\ wutiphol@mathstat.sci.tu.ac.th \\ ${ }^{2}$ Department of Mathematics and \\ Statistics, Faculty of Science and \\ Technology, Thammasat University \\ Rangsit Center, Pathumthani, 12121 , \\ Thailand \\ Full list of author information is \\ available at the end of the article
}

\begin{abstract}
In this paper, we establish new fixed point results for multi-valued operator on a cone metric space with respect to a solid cone by using the idea of the generalized Hausdorff distance due to Cho and Bae (Fixed Point Theory Appl. 2011:87, 2011). We also furnish some interesting examples which support our main theorems and give many results as corollaries of our result. As applications of our results, we obtain fixed point results for the multi-valued contraction operators in cone metric spaces endowed with graph.
\end{abstract}

MSC: $47 \mathrm{H} 10 ; 54 \mathrm{H} 25$

Keywords: $\alpha$-admissible multi-valued operator; cone metric space; directed graph; the generalized Hausdorff distance

\section{Introduction}

In 2007, Huang and Zhang [1] re-introduced the concept of cone metric space which is replacing the set of real numbers (as the co-domain of a metric) by an ordered Banach space. They described the convergence sequence in this space via interior points of the cone and also introduced the concept of completeness. By using these concepts, they proved some fixed point theorem for contractive type mappings which is a generalization of Banach's contraction principle. Afterward, many authors studied and extended fixed point theorems in cone metric spaces (see [2-4] and references therein).

In 2011, Janković et al. [5] showed that most of the fixed point results for mappings satisfying a contractive type condition in cone metric spaces with normal cone can be reduced to the corresponding results from metric space theory. They also give some examples and showed that fixed point theorems from ordinary metric spaces cannot be applied in the setting of cone metric spaces whenever the cone is non-normal. In fact, the fixed point problem in the setting of cone metric spaces is appropriate only in the case when the underlying cone is non-normal with non-empty interior (such cones are called solid) because the results concerning fixed points and other results in this case cannot be proved by reducing to metric spaces. So fixed point results in this trend are still of interest and importance in some ways.

On the other hand, a study of fixed point for multi-valued (set-valued) operators was originally initiated by von Neumann [6] in the study of game theory. In 1969, the development of geometric fixed point theory for a multi-valued operator via the concept of Haus- 
dorff distance was initiated with the work of Nadler [7], usually referred to as Nadler's contraction principle. Many authors in [8-11] proved fixed point theorems for multi-valued operators in cone metric spaces which are generalizations of classical Nadler's contraction principle.

Recently, Cho and Bae [12] first introduced the concept of the generalized Hausdorff distance operator in cone metric spaces and initially studied fixed point results for a multivalued operator via such a concept in cone metric spaces. Most recently, Kutbi et al. [13] established fixed point results for multi-valued operator under the generalized contractive condition via the generalized Hausdorff distance operator of Cho and Bae [12].

In this paper, we establish new fixed point results for multi-valued operator by using the idea of generalized Hausdorff distance in the context of cone metric spaces with respect to a solid cone. Our results unify, generalize, and complement results of Kutbi et al. [13] and many results from the literature. Also, we furnish some interesting examples which support our main theorems. As an application, we analyze the fixed points for the multivalued contraction operators in cone metric spaces endowed with a graph.

\section{Preliminaries}

Throughout this paper, we denote by $\mathbb{N}, \mathbb{R}_{+}$and $\mathbb{R}$ the sets of positive integers, nonnegative real numbers and real numbers, respectively.

Now, we recall some definitions and lemmas in cone metric spaces and the notion of the generalized Hausdorff distance operator of Cho and Bae [12], which will be required in the sequel.

Let $\left(\mathbb{E},\|\cdot\|_{\mathbb{E}}\right)$ be a real Banach space and $\theta$ be a zero element in $\mathbb{E}$. A subset $P$ of $\mathbb{E}$ is called a cone if the following conditions are satisfied:

$\left(C_{1}\right) P$ is non-empty closed and $P \neq\{\theta\}$;

$\left(C_{2}\right) a x+b y \in P$ for all $x, y \in P$ and $a, b \geq 0$;

$\left(C_{3}\right) P \cap(-P)=\{\theta\}$.

For a given cone $P \subseteq \mathbb{E}$, we define a partial ordering $\preceq$ with respect to $P$ by $x \preceq y$ if and only if $y-x \in P$ and $x \prec y$ stands for $x \preceq y$ and $x \neq y$, while $x \ll y$ stands for $y-x \in \operatorname{int}(P)$, where int $(P)$ denotes the interior of $P$.

The cone $P$ is said to be normal if there exists a real number $K>0$ such that for all $x, y \in E$,

$$
\theta \preceq x \preceq y \quad \Longrightarrow \quad\|x\|_{E} \leq K\|y\|_{E} .
$$

The least positive number $K$ satisfying the above statement is called the normal constant of $P$. In 2008, Rezapour and Hamlbarani [14] showed that there are no normal cones with normal constant $K<1$.

Definition 2.1 ([1]) Let $X$ be a non-empty set and $P$ be a cone in real Banach space $\mathbb{E}$. If a function $d: X \times X \rightarrow \mathbb{E}$ satisfies the following conditions:

$\left(\mathrm{CM}_{1}\right) \theta \preceq d(x, y)$ for all $x, y \in X$ and $d(x, y)=\theta$ if and only if $x=y$;

$\left(\mathrm{CM}_{2}\right) d(x, y)=d(y, x)$ for all $x, y \in X$;

$\left(\mathrm{CM}_{3}\right) d(x, z) \preceq d(x, y)+d(y, z)$ for all $x, y, z \in X$,

then $d$ is called a cone metric on $X$ and $(X, d)$ is called a cone metric space. 
Definition $2.2([1])$ Let $(X, d)$ be a cone metric space with cone $P$ in real Banach space $\mathbb{E}$, $\left\{x_{n}\right\}$ be a sequence in $X$ and $x \in X$.

1. If for every $c \in \mathbb{E}$ with $\theta \ll c$, there is $N \in \mathbb{N}$ such that $d\left(x_{n}, x\right) \ll c$ for all $n \geq N$, then $\left\{x_{n}\right\}$ is said to converge to $x$. This limit is denoted by $\lim _{n \rightarrow \infty} x_{n}=x$ or $x_{n} \rightarrow x$ as $n \rightarrow \infty$.

2. If for every $c \in \mathbb{E}$ with $\theta \ll c$, there is $N \in \mathbb{N}$ such that $d\left(x_{n}, x_{m}\right) \ll c$ for all $n, m>N$, then $\left\{x_{n}\right\}$ is called a Cauchy sequence in $X$.

3. If every Cauchy sequence in $X$ is convergent in $X$, then $(X, d)$ is called a complete cone metric space.

Remark 2.1 The cone metric is not continuous in the general case, i.e., from $x_{n} \rightarrow x$, $y_{n} \rightarrow y$ as $n \rightarrow \infty$ it need not follow that $d\left(x_{n}, y_{n}\right) \rightarrow d(x, y)$ as $n \rightarrow \infty$. However, if $(X, d)$ is a cone metric space with a normal cone $P$, then the cone metric $d$ is continuous (see Lemma 5 in [1]).

Definition 2.3 ([1]) Let $(X, d)$ be a cone metric space. A subset $A \subseteq X$ is called closed if for any sequence $\left\{x_{n}\right\}$ in $A$ convergent to $x$, we have $x \in A$.

Lemma 2.1 ([5]) Let $(X, d)$ be a cone metric space (particularly when dealing with cone metric spaces in which the cone need not be normal). Then the following properties hold:

$\left(\mathrm{PT}_{1}\right)$ If $u \preceq v$ and $v \ll w$, then $u \ll w$.

$\left(\mathrm{PT}_{2}\right)$ If $u \ll v$ and $v \preceq w$, then $u \ll w$.

$\left(\mathrm{PT}_{3}\right)$ If $u \ll v$ and $v \ll w$, then $u \ll w$.

$\left(\mathrm{PT}_{4}\right)$ If $\theta \preceq u \ll c$ for each $c \in \operatorname{int}(P)$, then $u=\theta$.

$\left(\mathrm{PT}_{5}\right)$ If $a \preceq b+c$, for each $c \in \operatorname{int}(P)$, then $a \preceq b$.

$\left(\mathrm{PT}_{6}\right)$ If $c \in \operatorname{int}(P)$ and $\left\{a_{n}\right\}$ is a sequence in $\mathbb{E}$ such that $\theta \preceq a_{n}$ for all $n \in \mathbb{N}$ and $a_{n} \rightarrow \theta$ as $n \rightarrow \infty$, then there exists $N \in \mathbb{N}$ such that for all $n \geq N$, we have $a_{n} \ll c$.

For a cone metric space $(X, d)$, denote

$$
\begin{aligned}
& N(X):=\{A: A \text { is non-empty subset of } X\}, \\
& C B(X):=\{A \in N(X): A \text { is closed and bounded }\}, \\
& s(p):=\{q \in \mathbb{E}: p \preceq q\} \text { for } p \in \mathbb{E},
\end{aligned}
$$

and

$$
s(a, B):=\bigcup_{b \in B} s(d(a, b)) \quad \text { for } a \in X \text { and } B \in C B(X) .
$$

For $A, B \in C B(X)$ we denote the generalized Hausdorff distance induced by $d$ (see [12]) by the following notation:

$$
s(A, B):=\left(\bigcap_{a \in A} s(a, B)\right) \cap\left(\bigcap_{b \in B} s(b, A)\right) .
$$

The following remarks are found in [12]. 
Remark 2.2 Let $(X, d)$ be a cone metric space. The functional $H: C B(X) \times C B(X) \rightarrow$ $[0, \infty)$ defined by

$$
H(A, B)=\inf s(A, B)
$$

for all $A, B \in C B(X)$, is the Hausdorff distance induced by $d$.

Remark 2.3 Let $(X, d)$ be a cone metric space. Then $s(\{a\},\{b\})=s(d(a, b))$ for all $a, b \in X$.

Lemma 2.2 ([12]) Let $(X, d)$ be a cone metric space with cone $P$ in real Banach space $\mathbb{E}$.

$\left(\mathrm{L}_{1}\right)$ Let $p, q \in \mathbb{E}$. If $p \preceq q$, then $s(q) \subseteq s(p)$.

$\left(\mathrm{L}_{2}\right)$ Let $x \in X$ and $A \in N(X)$. If $\theta \in s(x, A)$, then $x \in A$.

$\left(\mathrm{L}_{3}\right)$ Let $q \in P$ and $A, B \in C B(X)$. If $q \in s(A, B)$, then $q \in s(a, B)$ for all $a \in A$ and $q \in s(A, b)$ for all $b \in B$.

$\left(\mathrm{L}_{4}\right)$ Let $q \in P$ and let $\lambda \geq 0$, then $\lambda s(q) \subseteq s(\lambda q)$.

In 2012, Samet et al. [15] introduced the idea of an $\alpha$-admissible mapping and proved a fixed point theorem for a single valued mapping by using this concept. They showed that these results can be utilized to derive fixed point theorems in partially ordered spaces and also applied the main results to ordinary differential equations. Afterward, Asl et al. [16] introduced the concept of $\alpha_{*}$-admissible operator, which is multi-valued version of the $\alpha$-admissible mapping provided in [15].

Definition 2.4 ([16]) Let $X$ be a non-empty set, $F: X \rightarrow 2^{X}$, where $2^{X}$ is a collection of non-empty subset of $X$ and $\alpha: X \times X \rightarrow[0, \infty)$ be a mapping. We say that $F$ is $\alpha_{*-}$ admissible if the following condition holds:

$$
\text { for } x, y \in X \text { for which } \alpha(x, y) \geq 1 \quad \Longrightarrow \quad \alpha_{*}(F x, F y) \geq 1 \text {, }
$$

where $\alpha_{*}(F x, F y):=\inf \{\alpha(a, b) \mid a \in F x, b \in F y\}$.

Recently, Mohammadi et al. [17] extended the concept of $\alpha_{*}$-admissible operator to $\alpha$ admissible operator as follows.

Definition 2.5 ([17]) Let $X$ be a non-empty set, $F: X \rightarrow 2^{X}$, where $2^{X}$ is a collection of non-empty subset of $X$ and $\alpha: X \times X \rightarrow[0, \infty)$ be a mapping. We say that $F$ is $\alpha$-admissible whenever for each $x \in X$ and $y \in F x$ with $\alpha(x, y) \geq 1$, we have $\alpha(y, z) \geq 1$ for all $z \in F y$.

Remark 2.4 If $F$ is $\alpha_{*}$-admissible, then $F$ is also $\alpha$-admissible.

\section{Fixed point results for multi-valued operators}

In the following, we always suppose that $\Psi$ be a family of functions $\psi: P \rightarrow P$ such that

(i) $\psi(\theta)=\theta$ and $\theta \prec \psi(t) \prec t$ for $t \in P-\{\theta\}$,

(ii) $\psi(t) \ll t$ for all $\theta \ll t$,

(iii) $\lim _{n \rightarrow \infty} \psi^{n}(t)=\theta$ for every $t \in P-\{\theta\}$,

(iv) $\psi$ is a strictly increasing function, i.e., $\psi(a) \prec \psi(b)$ whenever $a \prec b$. 
Remark 3.1 From (i), we obtain $\psi(t) \preceq t$ for all $t \in P$.

Definition 3.1 Let $(X, d)$ be a cone metric space with cone $P$ in real Banach space $\mathbb{E}$, $\psi: P \rightarrow P$ and $\alpha: X \times X \rightarrow[0, \infty)$ be two given mappings. The multi-valued operator $F: X \rightarrow C B(X)$ is said to be an $\alpha-\psi$-contraction if

$$
\psi(d(x, y)) \in \alpha(x, y) s(F x, F y)
$$

for all $x, y \in X$,

Theorem 3.1 Let $(X, d)$ be a complete cone metric space with solid (normal or non-normal) cone $P$ in real Banach space $\mathbb{E}, \alpha: X \times X \rightarrow[0, \infty)$ be a given mapping, $\psi \in \Psi$ and $F: X \rightarrow$ $C B(X)$ be an $\alpha-\psi$-contraction multi-valued operator. Suppose that the following conditions hold:

(a) $F$ is $\alpha$-admissible operator;

(b) there exist $x_{0} \in X$ and $x_{1} \in F x_{0}$ such that $\alpha\left(x_{0}, x_{1}\right) \geq 1$;

(c) if $\left\{x_{n}\right\}$ is a sequence in $X$ such that $\alpha\left(x_{n}, x_{n+1}\right) \geq 1$ for all $n \in \mathbb{N}$ and $x_{n} \rightarrow u$ as $n \rightarrow \infty$, then $\alpha\left(x_{n}, u\right) \geq 1$ for all $n \in \mathbb{N}$.

Then there exists a point $x^{*} \in X$ such that $x^{*} \in F x^{*}$, that is, $F$ has a fixed point in $X$.

Proof We start from $x_{0}$ and $x_{1} \in F x_{0}$ in (b). Since $F$ is an $\alpha$ - $\psi$-contraction multi-valued operator, we have

$$
\psi\left(d\left(x_{0}, x_{1}\right)\right) \in \alpha\left(x_{0}, x_{1}\right) s\left(F x_{0}, F x_{1}\right) .
$$

Using Lemma 2.2( $\left.\mathrm{L}_{3}\right)$, we get

$$
\psi\left(d\left(x_{0}, x_{1}\right)\right) \in \alpha\left(x_{0}, x_{1}\right) s\left(x_{1}, F x_{1}\right) .
$$

By definition of $s\left(x_{1}, F x_{1}\right)$, we can take $x_{2} \in F x_{1}$ such that

$$
\psi\left(d\left(x_{0}, x_{1}\right)\right) \in \alpha\left(x_{0}, x_{1}\right) s\left(d\left(x_{1}, x_{2}\right)\right) .
$$

By Lemma 2.2( $\left.\mathrm{L}_{4}\right)$, we have

$$
\psi\left(d\left(x_{0}, x_{1}\right)\right) \in \alpha\left(x_{0}, x_{1}\right) s\left(d\left(x_{1}, x_{2}\right)\right) \subseteq s\left(\alpha\left(x_{0}, x_{1}\right) d\left(x_{1}, x_{2}\right)\right) .
$$

This implies that

$$
\alpha\left(x_{0}, x_{1}\right) d\left(x_{1}, x_{2}\right) \preceq \psi\left(d\left(x_{0}, x_{1}\right)\right) .
$$

Now, we have

$$
d\left(x_{1}, x_{2}\right) \preceq \alpha\left(x_{0}, x_{1}\right) d\left(x_{1}, x_{2}\right) \preceq \psi\left(d\left(x_{0}, x_{1}\right)\right) .
$$

It follows from $F$ being an $\alpha-\psi$-contraction multi-valued operator that

$$
\psi\left(d\left(x_{1}, x_{2}\right)\right) \in \alpha\left(x_{1}, x_{2}\right) s\left(F x_{1}, F x_{2}\right) .
$$


From Lemma $2.2\left(\mathrm{~L}_{3}\right)$, we obtain

$$
\psi\left(d\left(x_{1}, x_{2}\right)\right) \in \alpha\left(x_{1}, x_{2}\right) s\left(x_{2}, F x_{2}\right) .
$$

By definition of $s\left(x_{2}, F x_{2}\right)$, we can choose $x_{3} \in F x_{2}$ such that

$$
\psi\left(d\left(x_{1}, x_{2}\right)\right) \in \alpha\left(x_{1}, x_{2}\right) s\left(d\left(x_{2}, x_{3}\right)\right) .
$$

From Lemma 2.2( $\left(\mathrm{L}_{4}\right)$, we obtain

$$
\psi\left(d\left(x_{1}, x_{2}\right)\right) \in \alpha\left(x_{1}, x_{2}\right) s\left(d\left(x_{2}, x_{3}\right)\right) \subseteq s\left(\alpha\left(x_{1}, x_{2}\right) d\left(x_{2}, x_{3}\right)\right)
$$

and hence

$$
\alpha\left(x_{1}, x_{2}\right) d\left(x_{2}, x_{3}\right) \preceq \psi\left(d\left(x_{1}, x_{2}\right)\right) .
$$

Since $x_{1} \in F x_{0}, x_{2} \in F x_{1}, \alpha\left(x_{0}, x_{1}\right) \geq 1$ and $F$ is $\alpha$-admissible operator, we have $\alpha\left(x_{1}, x_{2}\right) \geq 1$. Therefore,

$$
d\left(x_{2}, x_{3}\right) \preceq \alpha\left(x_{1}, x_{2}\right) d\left(x_{2}, x_{3}\right) \preceq \psi\left(d\left(x_{1}, x_{2}\right)\right) .
$$

Inductively, we can construct a sequence $\left\{x_{n}\right\}$ in $X$ such that

$$
\begin{aligned}
& x_{n} \in F x_{n-1}, \\
& d\left(x_{n}, x_{n+1}\right) \preceq \psi\left(d\left(x_{n-1}, x_{n}\right)\right)
\end{aligned}
$$

and

$$
\alpha\left(x_{n-1}, x_{n}\right) \geq 1
$$

for all $n \in \mathbb{N}$. If there exists $n^{*} \in \mathbb{N}$ such that $x_{n^{*}-1}=x_{n^{*}}$, then $x_{n^{*}-1}$ is a fixed point of $F$. This finishes the proof. Therefore, we may assume that $x_{n-1} \neq x_{n}$ for all $n \in \mathbb{N}$. From (3.3), we obtain

$$
d\left(x_{n}, x_{n+1}\right) \preceq \psi\left(d\left(x_{n-1}, x_{n}\right)\right) \preceq \cdots \preceq \psi^{n}\left(d\left(x_{0}, x_{1}\right)\right)
$$

for all $n \in \mathbb{N}$. Since $\psi \in \Psi$, we have $\psi^{n}\left(d\left(x_{0}, x_{1}\right)\right) \rightarrow \theta$ as $n \rightarrow \infty$.

Fix $c \in \mathbb{E}$ such that $\theta \ll c$. Using the property of $\psi$, we get $\theta \ll c-\psi(c)$. By $\left(\mathrm{PT}_{6}\right)$, there is $N \in \mathbb{N}$ such that $\psi^{n}\left(d\left(x_{0}, x_{1}\right)\right) \ll c-\psi(c)$ and $\psi^{n}\left(d\left(x_{0}, x_{1}\right)\right) \ll c$ for $n \geq N$. Therefore, $d\left(x_{n}, x_{n+1}\right) \ll c-\psi(c)$ and $d\left(x_{n}, x_{n+1}\right) \ll c$ for $n \geq N$. For fixed $m>n \geq N$, we have

$$
\begin{aligned}
d\left(x_{n}, x_{n+2}\right) & \preceq d\left(x_{n}, x_{n+1}\right)+d\left(x_{n+1}, x_{n+2}\right) \ll c-\psi(c)+\psi\left(d\left(x_{n}, x_{n+1}\right)\right) \\
& \ll c-\psi(c)+\psi(c)=c, \\
d\left(x_{n}, x_{n+3}\right) & \preceq d\left(x_{n}, x_{n+1}\right)+d\left(x_{n+1}, x_{n+3}\right) \ll c-\psi(c)+\psi\left(d\left(x_{n}, x_{n+2}\right)\right) \\
& \ll c-\psi(c)+\psi(c)=c,
\end{aligned}
$$




$$
\begin{aligned}
& \\
d\left(x_{n}, x_{m}\right) & \preceq d\left(x_{n}, x_{n+1}\right)+d\left(x_{n+1}, x_{m}\right) \ll c-\psi(c)+\psi\left(d\left(x_{n}, x_{m-1}\right)\right) \\
& \ll c-\psi(c)+\psi(c)=c .
\end{aligned}
$$

This shows that $\left\{x_{n}\right\}$ is a Cauchy sequence. By the completeness of $X$, we get $x_{n} \rightarrow u$ for some $u \in X$. Using (3.4) and (c), we obtain $\alpha\left(x_{n}, u\right) \geq 1$ for all $n \in \mathbb{N}$. Now, we have

$$
\psi\left(d\left(x_{n}, u\right)\right) \in \alpha\left(x_{n}, u\right) s\left(F x_{n}, F u\right)
$$

for all $n \in \mathbb{N}$. By Lemma $2.2\left(\mathrm{~L}_{3}\right)$, we have

$$
\psi\left(d\left(x_{n}, u\right)\right) \in \alpha\left(x_{n}, u\right) s\left(x_{n+1}, F u\right)
$$

for all $n \in \mathbb{N}$. Therefore, we can choose $v_{n} \in F u$ such that

$$
\psi\left(d\left(x_{n}, u\right)\right) \in \alpha\left(x_{n}, u\right) s\left(d\left(x_{n+1}, v_{n}\right)\right)
$$

for all $n \in \mathbb{N}$. Using Lemma $2.2\left(\mathrm{~L}_{4}\right)$, we get

$$
\psi\left(d\left(x_{n}, u\right)\right) \in \alpha\left(x_{n}, u\right) s\left(d\left(x_{n+1}, v_{n}\right)\right) \subseteq s\left(\alpha\left(x_{n}, u\right) d\left(x_{n+1}, v_{n}\right)\right)
$$

and so

$$
\alpha\left(x_{n}, u\right) d\left(x_{n+1}, v_{n}\right) \preceq \psi\left(d\left(x_{n}, u\right)\right)
$$

for all $n \in \mathbb{N}$. Therefore, we have

$$
d\left(x_{n+1}, v_{n}\right) \preceq \alpha\left(x_{n}, u\right) d\left(x_{n+1}, v_{n}\right) \preceq \psi\left(d\left(x_{n}, u\right)\right) \preceq d\left(x_{n}, u\right)
$$

for all $n \in \mathbb{N}$. Since $x_{n} \rightarrow u$ as $n \rightarrow \infty$, for a given $c \in \mathbb{E}$ with $\theta \ll c$, there is $N^{\prime} \in \mathbb{N}$ such that

$$
d\left(u, x_{n}\right) \ll \frac{c}{2}
$$

for all $n \geq N^{\prime}$. It follows that

$$
\begin{aligned}
d\left(u, v_{n}\right) & \preceq d\left(u, x_{n+1}\right)+d\left(x_{n+1}, v_{n}\right) \\
& \preceq d\left(u, x_{n+1}\right)+d\left(x_{n}, u\right) \\
& \ll \frac{c}{2}+\frac{c}{2} \\
& =c
\end{aligned}
$$

for all $n \geq N^{\prime}$. Hence, we have $\lim _{n \rightarrow \infty} v_{n}=u$. Since $F u$ is closed and $v_{n} \in F u$ for all $n \in \mathbb{N}$, we obtain $u \in F u$. This implies that $u$ is a fixed point of $F$. This completes the proof.

Now we give an example to support Theorem 3.1. 
Example 3.2 Let $\mathbb{E}=C_{\mathbb{R}}^{1}[0,1]:=\{f:[0,1] \rightarrow \mathbb{R}: f$ have a continuous first ordered derivative on $[0,1]\}$ be a real Banach space with norm $\|\cdot\|_{\mathbb{E}}$ defined by

$$
\|f\|_{\mathbb{E}}=\sup _{t \in[0,1]}|f(t)|+\sup _{t \in[0,1]}\left|f^{\prime}(t)\right|
$$

for each $f \in \mathbb{E}$, where $\theta:=f_{0} \in \mathbb{E}$ which is defined by $f_{0}(t)=0$ for all $t \in[0,1]$. Define cone $P$ in $\mathbb{E}$ by

$$
P:=\{g \in \mathbb{E}: g(t) \geq 0 \text { for all } t \in[0,1]\} .
$$

This cone is solid but non-normal (see [18]).

Let $X=[0,10]$ and define cone metric $d: X \times X \rightarrow \mathbb{E}$ for each $x, y \in X$ as follows:

$$
d(x, y)(t)=|x-y| e^{t}
$$

for all $t \in[0,1]$. Define a mapping $\alpha: X \times X \rightarrow[0, \infty)$ and a multi-valued operator $F: X \rightarrow$ $C B(X)$ by

$$
\alpha(x, y)= \begin{cases}x^{2}+y^{2}+1, & x, y \in[0,1] \\ 0, & \text { otherwise }\end{cases}
$$

and

$$
F x= \begin{cases}\left\{\frac{x}{6}\right\}, & x \in[0,1], \\ {\left[\frac{10 x-10}{9}, x\right],} & \text { otherwise. }\end{cases}
$$

Now we show that $F$ is an $\alpha-\psi$-contraction multi-valued operator with $\psi: P \rightarrow P$ define by $\psi(f)=\frac{1}{2} f$ for all $f \in P$. For $x, y \in[0,1]$, we obtain

$$
\begin{aligned}
\alpha(x, y) d\left(\frac{x}{6}, \frac{y}{6}\right)(t) & =\left(x^{2}+y^{2}+1\right)\left|\frac{x-y}{6}\right| e^{t} \\
& \leq(1+1+1)\left|\frac{x-y}{6}\right| e^{t} \\
& =\frac{1}{2}|x-y| e^{t} \\
& =(\psi(d(x, y)))(t),
\end{aligned}
$$

which implies that $\psi(d(x, y)) \in \alpha(x, y) s(F x, F y)$. Otherwise, it is easy to see that $\psi(d(x, y)) \in$ $\alpha(x, y) s(F x, F y)$. Therefore, $F$ is an $\alpha-\psi$-contraction multi-valued operator.

Next, we show that our results in this paper can be used for this case. It is easy to see that $F$ is an $\alpha$-admissible operator and there exist $x_{0}=1$ and $x_{1}=1 / 6 \in F x_{0}$ such that $\alpha\left(x_{0}, x_{1}\right)=\alpha(1,1 / 6) \geq 1$. Also, we see that condition (c) in Theorem 3.1 holds. Therefore, all the conditions of Theorem 3.1 are satisfied and so $F$ has a fixed point.

Corollary 3.3 ([13]) Let $(X, d)$ be a complete cone metric space with solid (normal or nonnormal) cone $P$ in real Banach space $\mathbb{E}, \alpha: X \times X \rightarrow[0, \infty)$ be a given mapping, $\psi \in \Psi$ and 
$F: X \rightarrow C B(X)$ be an $\alpha$ - $\psi$-contraction multi-valued operator. Suppose that the following conditions hold:

(a) $F$ is $\alpha_{*}$-admissible operator;

(b) there exist $x_{0} \in X$ and $x_{1} \in F x_{0}$ such that $\alpha\left(x_{0}, x_{1}\right) \geq 1$;

(c) if $\left\{x_{n}\right\}$ is a sequence in $X$ such that $\alpha\left(x_{n}, x_{n+1}\right) \geq 1$ for all $n \in \mathbb{N}$ and $x_{n} \rightarrow u$ as $n \rightarrow \infty$, then $\alpha\left(x_{n}, u\right) \geq 1$ for all $n \in \mathbb{N}$.

Then there exists a point $x^{*} \in X$ such that $x^{*} \in F x^{*}$, that is, $F$ has a fixed point in $X$.

Proof We can prove this result by using Theorem 3.1 and Remark 2.4.

Corollary 3.4 Let $(X, d)$ be a complete cone metric space with solid (normal or nonnormal) cone $P$ in real Banach space $\mathbb{E}, \alpha: X \times X \rightarrow[0, \infty)$ be a given mapping and $F: X \rightarrow C B(X)$ be multi-valued operator such that

$$
k d(x, y) \in \alpha(x, y) s(F x, F y)
$$

for all $x, y \in X$, where $k \in[0,1)$. Suppose that the following conditions hold:

(a) $F$ is $\alpha$-admissible operator;

(b) there exist $x_{0} \in X$ and $x_{1} \in F x_{0}$ such that $\alpha\left(x_{0}, x_{1}\right) \geq 1$;

(c) if $\left\{x_{n}\right\}$ is a sequence in $X$ such that $\alpha\left(x_{n}, x_{n+1}\right) \geq 1$ for all $n \in \mathbb{N}$ and $x_{n} \rightarrow u$ as $n \rightarrow \infty$, then $\alpha\left(x_{n}, u\right) \geq 1$ for all $n \in \mathbb{N}$.

Then there exists a point $x^{*} \in X$ such that $x^{*} \in F x^{*}$, that is, $F$ has a fixed point in $X$.

Proof By taking $\psi(t)=k t$ for all $t \in P$ in Theorem 3.1, we get this result.

Corollary 3.5 Let $(X, d)$ be a complete cone metric space with solid (normal or nonnormal) cone $P$ in real Banach space $\mathbb{E}, \psi \in \Psi$ and $F: X \rightarrow C B(X)$ be multi-valued operator such that

$$
\psi(d(x, y)) \in s(F x, F y)
$$

for all $x, y \in X$. Then there exists a point $x^{*} \in X$ such that $x^{*} \in F x^{*}$, that is, $F$ has a fixed point in $X$.

Proof By taking $\alpha(x, y)=1$ for all $x, y \in X$ in Theorem 3.1, we get this result.

Corollary 3.6 Let $(X, d)$ be a complete cone metric space with solid (normal or nonnormal) cone $P$ in real Banach space $\mathbb{E}$ and $F: X \rightarrow C B(X)$ be multi-valued operator such that

$$
k d(x, y) \in s(F x, F y)
$$

for all $x, y \in X$, where $k \in[0,1)$. Then there exists a point $x^{*} \in X$ such that $x^{*} \in F x^{*}$, that is, $F$ has a fixed point in $X$.

Proof By taking $\psi(t)=k t$ for all $t \in P$ and $\alpha(x, y)=1$ for all $x, y \in X$ in Theorem 3.1, we get this result.

By Remark 2.2, we get the following results. 
Corollary 3.7 Let $(X, d)$ be a complete metric space, $\alpha: X \times X \rightarrow[0, \infty)$ be a given mapping and $F: X \rightarrow C B(X)$ be multi-valued operator such that

$$
\alpha(x, y) H(F x, F y) \leq k d(x, y)
$$

for all $x, y \in X$, where $k \in[0,1)$. Suppose that the following conditions hold:

(a) $F$ is $\alpha$-admissible operator;

(b) there exist $x_{0} \in X$ and $x_{1} \in F x_{0}$ such that $\alpha\left(x_{0}, x_{1}\right) \geq 1$;

(c) if $\left\{x_{n}\right\}$ is a sequence in $X$ such that $\alpha\left(x_{n}, x_{n+1}\right) \geq 1$ for all $n \in \mathbb{N}$ and $x_{n} \rightarrow u$ as $n \rightarrow \infty$, then $\alpha\left(x_{n}, u\right) \geq 1$ for all $n \in \mathbb{N}$.

Then there exists a point $x^{*} \in X$ such that $x^{*} \in F x^{*}$, that is, $F$ has a fixed point in $X$.

Corollary 3.8 ([7]) Let $(X, d)$ be a complete metric space and $F: X \rightarrow C B(X)$ be multivalued operator such that

$$
H(F x, F y) \leq k d(x, y)
$$

for all $x, y \in X$, where $k \in[0,1)$. Then there exists a point $x^{*} \in X$ such that $x^{*} \in F x^{*}$, that is, $F$ has a fixed point in $X$.

Next, we give a lemma which is useful to prove the second main result. Moreover, this lemma is also a tool for analyzing fixed point results in a directed graph in Section 4.

Lemma 3.9 Let $(X, d)$ be a cone metric space with cone $P$ in real Banach space $\mathbb{E}, \alpha$ : $X \times X \rightarrow[0, \infty)$ be a given mapping. If $\alpha$ has transitive property (i.e., if $\alpha(x, y) \geq 1$ and $\alpha(y, z) \geq 1$, then $\alpha(x, z) \geq 1)$, then the following conditions are equivalent:

(c) if $\left\{x_{n}\right\}$ is a sequence in $X$ such that $\alpha\left(x_{n}, x_{n+1}\right) \geq 1$ for all $n \in \mathbb{N}$ and $x_{n} \rightarrow u$ as $n \rightarrow \infty$, then $\alpha\left(x_{n}, u\right) \geq 1$ for all $n \in \mathbb{N}$;

(c') if $\left\{x_{n}\right\}$ is a sequence in $X$ such that $\alpha\left(x_{n}, x_{n+1}\right) \geq 1$ for all $n \in \mathbb{N}$ and $x_{n} \rightarrow u$ as $n \rightarrow \infty$, then there is a subsequence $\left\{x_{k_{n}}\right\}$ with $\alpha\left(x_{k_{n}}, u\right) \geq 1$ for all $n \in \mathbb{N}$.

Proof It easy to see that $(\mathrm{c}) \Longrightarrow\left(\mathrm{c}^{\prime}\right)$. Now assume $\left(\mathrm{c}^{\prime}\right)$ holds and $\left\{x_{n}\right\}$ is as in (c). By transitivity, $\alpha\left(x_{n}, x_{m}\right) \geq 1$ if $n \leq m$. From ( $\left.\mathrm{c}^{\prime}\right)$, there is a subsequence $\left\{x_{k_{n}}\right\}$ such that $\alpha\left(x_{k_{n}}, u\right) \geq 1$ for $n \in \mathbb{N}$. Since $n \leq k_{n}$, we have $\alpha\left(x_{n}, x_{k_{n}}\right) \geq 1$. By transitivity, we have $\alpha\left(x_{n}, u\right) \geq 1$.

Using Lemma 3.9, we have the following result.

Corollary 3.10 Let $(X, d)$ be a complete cone metric space with solid (normal or nonnormal) cone $P$ in a real Banach space $\mathbb{E}, \alpha: X \times X \rightarrow[0, \infty)$ be a mapping satisfying transitive property, $\psi \in \Psi$ and $F: X \rightarrow C B(X)$ be an $\alpha$ - $\psi$-contraction multi-valued operator. Suppose that the following conditions hold:

(a) $F$ is $\alpha$-admissible operator;

(b) there exist $x_{0} \in X$ and $x_{1} \in F x_{0}$ such that $\alpha\left(x_{0}, x_{1}\right) \geq 1$;

(c') if $\left\{x_{n}\right\}$ is a sequence in $X$ such that $\alpha\left(x_{n}, x_{n+1}\right) \geq 1$ for all $n \in \mathbb{N}$ and $x_{n} \rightarrow u$ as $n \rightarrow \infty$, then there is a subsequence $\left\{x_{k_{n}}\right\}$ with $\alpha\left(x_{k_{n}}, u\right) \geq 1$ for all $n \in \mathbb{N}$.

Then there exists a point $x^{*} \in X$ such that $x^{*} \in F x^{*}$, that is, $F$ has a fixed point in $X$. 
Corollary 3.11 Let $(X, d)$ be a complete cone metric space with solid (normal or nonnormal) cone P in real Banach space $\mathbb{E}, \alpha: X \times X \rightarrow[0, \infty)$ be a mapping satisfying transitive property, $\psi \in \Psi$ and $F: X \rightarrow C B(X)$ be an $\alpha$ - $\psi$-contraction multi-valued operator. Suppose that the following conditions hold:

(a) $F$ is $\alpha_{*}$-admissible operator;

(b) there exist $x_{0} \in X$ and $x_{1} \in F x_{0}$ such that $\alpha\left(x_{0}, x_{1}\right) \geq 1$;

(c') if $\left\{x_{n}\right\}$ is a sequence in $X$ such that $\alpha\left(x_{n}, x_{n+1}\right) \geq 1$ for all $n \in \mathbb{N}$ and $x_{n} \rightarrow$ as $n \rightarrow \infty$, then there is a subsequence $\left\{x_{k_{n}}\right\}$ with $\alpha\left(x_{k_{n}}, u\right) \geq 1$ for all $n \in \mathbb{N}$.

Then there exists a point $x^{*} \in X$ such that $x^{*} \in F x^{*}$, that is, $F$ has a fixed point in $X$.

\section{Fixed point analysis with graph}

Throughout this section let $(X, d)$ be a cone metric space. A set $\{(x, x): x \in X\}$ is called a diagonal of the Cartesian product $X \times X$ and is denoted by $\Delta$. Consider a directed graph $G$ such that the set $V(G)$ of its vertices coincides with $X$ and the set $E(G)$ of its edges contains all loops, i.e., $\Delta \subseteq E(G)$. We assume $G$ has no parallel edges, so we can identify $G$ with the pair $(V(G), E(G))$. Moreover, we may treat $G$ as a weighted graph by assigning to each edge the distance between its vertices. If $x$ and $y$ are vertices in a graph $G$, then a path in $G$ from $x$ to $y$ of length $N(N \in \mathbb{N} \cup\{0\})$ is a sequence $\left\{x_{i}\right\}_{i=0}^{N}$ of $N+1$ vertices such that $x_{0}=x$, $x_{N}=y$ and $\left(x_{n-1}, x_{n}\right) \in E(G)$ for $i=1, \ldots, N$.

A graph $G$ is connected if there is a path between any two vertices. Also, $G$ is weakly connected if $\tilde{G}$ is connected, where $\tilde{G}$ is the undirected graph obtained from $G$ by ignoring the direction of the edges.

Recently, some results have appeared providing sufficient conditions for a single valued mapping and a multi-valued operator on some space which is endowed with a graph to be a Picard operator. The first result in this direction was given by Jachymski [19].

In this section, we give the existence of fixed point theorems for multi-valued operator in a cone metric space endowed with a graph under the generalized Hausdorff distance. Before presenting our results, we will introduce new definitions in a cone metric space endowed with a graph.

Definition 4.1 Let $(X, d)$ be a cone metric space (with cone $P$ in real Banach space $\mathbb{E}$ ) endowed with a graph $G$ and $F: X \rightarrow C B(X)$ be multi-valued operator. We say that $F$ weakly preserves edges of $G$ if for each $x \in X$ and $y \in F x$ with $(x, y) \in E(G)$ it is implied that $(y, z) \in E(G)$ for all $z \in F y$.

Definition 4.2 Let $(X, d)$ be a cone metric space (with cone $P$ in real Banach space $\mathbb{E}$ ) endowed with a graph $G$ and $\psi \in \Psi$. A multi-valued operator $F: X \rightarrow C B(X)$ is said to be a $\psi$-G-contraction if

$$
\psi(d(x, y)) \in s(F x, F y)
$$

for all $x, y \in X$ with $(x, y) \in E(G)$. If $\psi$ defined by $\psi(t)=k t$ for all $t \in P$, where $k \in[0,1)$, then $F$ is said to be a generalized Banach $G$-contraction.

Remark 4.1 Note that a Banach G-contraction in the sense of being multi-valued, i.e.,

$$
H(F x, F y) \preceq k d(x, y)
$$


for all $x, y \in X$ for which $(x, y) \in E(G)$, where $k \in[0,1)$, is a $\psi$-G-contraction by using Remark 2.2.

Example 4.1 Any mapping $F: X \rightarrow C B(X)$ defined by $F x=\{a\}$, where $a \in X$, is a $\psi$-Gcontraction for any graph $G$ with $V(G)=X$ and all $\psi \in \Psi$.

Example 4.2 Any mapping $F: X \rightarrow C B(X)$ is trivially a $\psi$-G-contraction, where $G=$ $(V(G), E(G))=(X, \Delta)$.

Definition 4.3 Let $(X, d)$ be a cone metric space (with cone $P$ in real Banach space $\mathbb{E}$ ) endowed with a graph $G$. We say that $X$ has the G-regular property if given $x \in X$ and sequence $\left\{x_{n}\right\}$ in $X$ such that $x_{n} \rightarrow x$ as $n \rightarrow \infty$ and $\left(x_{n}, x_{n+1}\right) \in E(G)$ for all $n \in \mathbb{N}$, then $\left(x_{n}, u\right) \in E(G)$ for all $n \in \mathbb{N}$.

Definition 4.4 Let $(X, d)$ be a cone metric space (with cone $P$ in real Banach space $\mathbb{E}$ ) endowed with a graph $G$. We say that $E(G)$ is quasi-ordered (or transitive) if $(x, y) \in E(G)$ and $(y, z) \in E(G)$ imply $(x, y) \in E(G)$ for all $x, y, z \in X$.

Here, we give two fixed point results for multi-valued operator in a cone metric space endowed with a graph.

Theorem 4.3 Let $(X, d)$ be a complete cone metric space (with cone P in real Banach space $\mathbb{E})$ endowed with a graph $G$ and $F: X \rightarrow C B(X)$ be a $\psi$-G-contraction. Suppose that the following conditions hold:

(A) F weakly preserves edges of $G$;

(B) there exist $x_{0} \in X$ and $x_{1} \in F x_{0}$ such that $\left(x_{0}, x_{1}\right) \in E(G)$;

(C) $X$ has the G-regular property.

Then there exists a point $x^{*} \in X$ such that $x^{*} \in F x^{*}$, that is, $F$ has a fixed point in $X$.

Proof Consider the mapping $\alpha: X \times X \rightarrow[0, \infty)$ defined by

$$
\alpha(x, y)= \begin{cases}1, & (x, y) \in E(G) ; \\ 0, & \text { otherwise. }\end{cases}
$$

Since $F$ is a $\psi-G$-contraction, we have, for all $x, y \in X$,

$$
\psi(d(x, y)) \in \alpha(x, y) s(F x, F y)
$$

This implies that $F$ satisfies (3.1). By construction of $\alpha$ and hypothesis (A), we see that $F$ is an $\alpha$-admissible operator. From condition (B) and the definition of $\alpha$, we get $\alpha\left(x_{0}, x_{1}\right) \geq 1$. Using the $G$-regular property of $X$, we find that condition (c) in Theorem 3.1 holds. Now all the hypotheses of Theorem 3.1 are satisfied and so the existence of the fixed point of $F$ follows from Theorem 3.1 .

Corollary 4.4 Let $(X, d)$ be a complete cone metric space (with cone P in real Banach space $\mathbb{E})$ endowed with a graph $G$ and $F: X \rightarrow C B(X)$ be a generalized Banach G-contraction. Suppose that the following conditions hold: 
(A) $F$ weakly preserves edges of $G$;

(B) there exist $x_{0} \in X$ and $x_{1} \in F x_{0}$ such that $\left(x_{0}, x_{1}\right) \in E(G)$;

(C) $X$ has the G-regular property.

Then there exists a point $x^{*} \in X$ such that $x^{*} \in F x^{*}$, that is, $F$ has a fixed point in $X$.

By Remark 2.2, we get the multi-valued version of Jachymski's result in [19].

Corollary 4.5 Let $(X, d)$ be a complete cone metric space (with cone $P$ in real Banach space $\mathbb{E})$ endowed with a graph $G$ and $F: X \rightarrow C B(X)$ be a multi-valued operator such that

$$
H(F x, F y) \preceq k d(x, y)
$$

for all $x, y \in X$ with $(x, y) \in E(G)$, where $k \in[0,1)$. Suppose that the following conditions hold:

(A) F weakly preserves edges of G;

(B) there exist $x_{0} \in X$ and $x_{1} \in F x_{0}$ such that $\left(x_{0}, x_{1}\right) \in E(G)$;

(C) $X$ has the G-regular property.

Then there exists a point $x^{*} \in X$ such that $x^{*} \in F x^{*}$, that is, $F$ has a fixed point in $X$.

Next, we give the fixed point result for a multi-valued operator in a connected graph.

Theorem 4.6 Let $(X, d)$ be a complete cone metric space (with cone $P$ in real Banach space $\mathbb{E})$ endowed with a connected graph $G$ and $F: X \rightarrow C B(X)$ be a $\psi$-G-contraction. Suppose that the following conditions hold:

(A) $F$ weakly preserves edges of $G$;

(B) there exist $x_{0} \in X$ and $x_{1} \in F x_{0}$ such that $\left(x_{0}, x_{1}\right) \in E(G)$;

$\left(C^{\prime}\right)$ if $u \in X$ and a sequence $\left\{x_{n}\right\}$ in $X$ are given such that $x_{n} \rightarrow u$ as $n \rightarrow \infty$ and $\left(x_{n}, x_{n+1}\right) \in$ $E(G)$ for all $n \in \mathbb{N}$, then there is a subsequence $\left\{x_{k_{n}}\right\}$ with $\left(x_{k_{n}}, u\right) \in E(G)$ for all $n \in \mathbb{N}$.

Then there exists a point $x^{*} \in X$ such that $x^{*} \in F x^{*}$, that is, $F$ has a fixed point in $X$.

Proof Since $G$ is a connected graph, $E(G)$ is a quasi-ordered. Consider the mapping $\alpha$ : $X \times X \rightarrow[0, \infty)$ defined as in the proof of Theorem 4.3. It follows from $E(G)$ being quasiordered that $\alpha$ has the transitive property. By Lemma 3.9, we note that condition $\left(C^{\prime}\right)$ is in equivalence to condition $(\mathrm{C})$ in Theorem 4.3. Thus we can prove this theorem similarly to the proof of Theorem 4.3 and consequently $F$ has a fixed point in $X$.

Corollary 4.7 Let $(X, d)$ be a complete cone metric space (with cone P in real Banach space $\mathbb{E})$ endowed with a connected graph $G$ and $F: X \rightarrow C B(X)$ be a generalized Banach $G$ contraction. Suppose that the following conditions hold:

(A) $F$ weakly preserves edges of $G$;

(B) there exist $x_{0} \in X$ and $x_{1} \in F x_{0}$ such that $\left(x_{0}, x_{1}\right) \in E(G)$;

(C') if $u \in X$ and a sequence $\left\{x_{n}\right\}$ in $X$ are given such that $x_{n} \rightarrow u$ as $n \rightarrow \infty$ and $\left(x_{n}, x_{n+1}\right) \in$ $E(G)$ for all $n \in \mathbb{N}$, then there is a subsequence $\left\{x_{k_{n}}\right\}$ with $\left(x_{k_{n}}, u\right) \in E(G)$ for all $n \in \mathbb{N}$.

Then there exists a point $x^{*} \in X$ such that $x^{*} \in F x^{*}$, that is, $F$ has a fixed point in $X$. 


\section{Competing interests}

The authors declare that they have no competing interests.

\section{Authors' contributions}

All authors contributed equally and significantly in writing this paper. All authors read and approved the final manuscript.

\section{Author details}

${ }^{1}$ Department of Mathematics, King Abdulaziz University, P.O. Box 80203, Jeddah, 21589, Saudi Arabia. ${ }^{2}$ Department of Mathematics and Statistics, Faculty of Science and Technology, Thammasat University Rangsit Center, Pathumthani, 12121, Thailand.

\section{Acknowledgements}

The authors thank the editor and the referees for their valuable and insightful comments and suggestions which improved greatly the quality of this paper. The first author gratefully acknowledges the support from the Deanship of Scientific Research (DSR) at King Abdulaziz University (KAU) during this research. The second author would like to thank the Thailand Research Fund and Thammasat University under Grant No. TRG5780013 for financial support during the preparation of this manuscript.

Received: 22 February 2014 Accepted: 26 June 2014 Published: 22 July 2014

\section{References}

1. Huang, LG, Zhang, X: Cone metric spaces and fixed point theorems of contractive mappings. J. Math. Anal. Appl. 332, 1468-1476 (2007)

2. Ilic, D, Pavlovic, V: Common fixed points for maps on cone metric space. J. Math. Anal. Appl. 341, 876-882 (2008)

3. Sintunavarat, W, Kumam, P: Common fixed points of $f$-weak contractions in cone metric spaces. Bull. Iran. Math. Soc. 38(2), 293-303 (2012)

4. Sintunavarat, W, Cho, YJ, Kumam, P: Common fixed point theorems for c-distance in ordered cone metric spaces. Comput. Math. Appl. 62, 1969-1978 (2011)

5. Janković, S, Kadelburg, Z, Radenović, S: On cone metric spaces: a survey. Nonlinear Anal. 74, 2591-2601 (2011)

6. von Neumann, J: Zur theorie der gesellschaftsspiele. Math. Ann. 100(1), 295-320 (1928)

7. Nadler, SB Jr.: Multi-valued contraction mappings. Pac. J. Math. 30, 475-488 (1969)

8. Kadelburg, Z, Radenovic, S: Some results on set-valued contractions in abstract metric spaces. Comput. Math. Appl. $62,342-350(2011)$

9. Klim, D, Wardowski, D: Dynamic processes and fixed points of set-valued nonlinear contractions in cone metric spaces. Nonlinear Anal. 71, 5170-5175 (2009)

10. Latif, A, Shaddad, FY: Fixed point results for multivalued maps in cone metric spaces. Fixed Point Theory Appl. 2010, Article ID 941371 (2010)

11. Wardowski, D: Endpoints and fixed points of set-valued contractions in cone metric spaces. Nonlinear Anal. 71, 512-516 (2009)

12. Cho, SH, Bae, JS: Fixed point theorems for multivalued maps in cone metric spaces. Fixed Point Theory Appl. 2011, 87 (2011)

13. Kutbi, MA, Ahmad, J, Azam, A: On fixed points of $\alpha-\psi$ contractive multi-valued mappings in cone metric spaces. Abstr. Appl. Anal. 2013, Article ID 313782 (2013)

14. Rezapour, S, Hamlbarani, R: Some notes on paper 'Cone metric spaces and fixed point theorems of contractive mappings'. J. Math. Anal. Appl. 345, 719-724 (2008)

15. Samet, B, Vetro, C, Vetro, P: Fixed point theorems for $\alpha$ - $\psi$-contractive type mappings. Nonlinear Anal. 75, 2154-2165 (2012)

16. Asl, JH, Rezapour, S, Shahzad, N: On fixed points of $\alpha-\psi$-contractive multifunctions. Fixed Point Theory Appl. 2012 , $212(2012)$

17. Mohammadi, B, Rezapour, S, Shahzad, N: Some results on fixed points of $\alpha$ - $\psi$-Ciric generalized multifunctions. Fixed Point Theory Appl. 2013, 24 (2013)

18. Vandergraft, JS: Newton's method for convex operators in partially ordered spaces. SIAM J. Numer. Anal. 4, 406-432 (1967)

19. Jachymski, J: The contraction principle for mappings on a metric space with a graph. Proc. Am. Math. Soc. 1(136), 1359-1373 (2008)

doi:10.1186/1687-1812-2014-142

Cite this article as: Kutbi and Sintunavarat: Fixed point analysis for multi-valued operators with graph approach by the generalized Hausdorff distance. Fixed Point Theory and Applications 2014 2014:142. 